\title{
Ecological Study on some Grasses Growing Naturally in the Deltaic Mediterranean Coast of Egypt
}

\author{
Ibrahim A. Mashaly ${ }^{*}$, Sekina M. Ayaad, Maha M. El-Shamy, Esraa A.Elsoudy \\ Botany Department, Faculty of Science, Mansoura University, Egypt
}

\begin{abstract}
The present study aims to investigate the floristic composition and vegetation analysis of the flora associated with some grasses growing naturally on the sand formations in the Deltaic Mediterranean coast of Egypt. The total number of the recorded plant species was 79 species ( 44 annuals and 35 perennials) belonging to 70 genera and 26 families. The life -form spectra of the recorded species comprised 44 therophytes, 13 cryptophytes, eight hemicryptophytes, seven chamaephytes and phanerphytes for each. Chorologically, the Saharo-Sindian element was represented by relatively high number of species (34), followed by Irano-Turanian (32 species), then Euro-Siberian (10 species) and Sudano-Zambezian ( 9 species). Based on the importance alue of each species, the classification of the recorded species in 36 sampled stands led to recognition of four vegetation groups (A-D). The characteristics dominant and codominant species of vegetation groups were Elymus farcatus and Calligonum polygonoides (A), Hordeum murinum (B), Stipagrostis lanata and Echinops spinosus (C) and Lycium schweinfurthii and Echinops spinosus (D). DCA diagram showed overlapping between the identified groups. CCA-biplot exhibited that sulfates, calcium, potassium, magnesium and calcium carbonate were the most effective soil variables controlling the abundance of identified vegetation groups.
\end{abstract}

Keywords: Grasses, Mediterranean coast, classification, ordination, soil.

\section{INTRODUCTION}

The Mediterranean coastal region of Egypt can be divided into three sections: 1) the western section (Mareotis coast) between Abu Qir at east to Sallum at west for about $550 \mathrm{~km}$ long, 2) the middle section (Deltaic coast) between Port- Said at east to Abu Qir at west for about $220 \mathrm{~km}$ long and 3) the eastern section (Sinai coast) between Rafah at east to Port-Said at west for about $200 \mathrm{~km}$ long. The vegetation of the coastal zone is considered the richest one among the phytogeographical regions of Egypt, where its flora represents more than $50 \%$ of the total flora of Egypt (Hassib,1951; Täckholm,1974).

The Deltaic Mediterranean coast can be distinguished into four main habitats:1) Sand formations (mounds, sheets and sand dunes), 2) Salt marshes (black barren, wet and dry), 3) Fertile sandy lands (cultivated and non-cultivated) and 4) Reed swamps. The sand formations are basically siliceous deposits mixed with clay and silt particles. The sand dunes are considered as the main geomorphic features especially in western part of this coast. These dunes can be classified into three types: mobile dunes, partial stabilized dunes and stabilized dunes (Mashaly, 2001\&2002; Mashaly et al. (2008). Floristically, Poaceae is considered as the richest family in the Deltaic Mediterranean coast, where it dominates all habitats of this area, especially the sand formation habitat type.

Grasses (constitute the largest and most valuable family among the flowering plants, with about 10000 species in the flora of Egypt; it includes 277 species (Boulos, 2009). Grasses occupy wide tracts of land and they are evenly distributed in all parts of the world. They occur in every soil, in all kinds of situation and under all climatic conditions. Although several families of plants supply the wants of man, the grass family exceeds all the others in the amount and the value of its products. The grasses growing in pasture land and the cereals grown all over the world are of more value to man and his domestic animals than all the other plants taken together (Rangachari and Tadulingam, 2007). Therefore, the selection of some natural grasses in the present study is required and urgent. Therefore, the main objective of the present investigation aims to provide insight on the floristic compition and vegetation analysis of flora associated with some grasses growing naturally in sand formations in the Delta Nile Coast of Egypt.

\section{The Study Area}

The sampled stands were distributed in the sand formations in the Deltaic Mediterranean coast of Egypt (Figure1).

The geologic and vegetation structures of the Nile Delta as a part of the northern Egypt has been subjected to the same geologic events that affected the whole region during its Pre-Miocene geological history. The major elements of geomorphology of Deltaic section were given by Sestini (1976). These elements comprise two main geomorphological components: coastal and deltaic. Mashaly (1987) stated that the Deltaic coastal section is characterized by morphological structure basically different from that to the west of Abu-Qir Bay (Mareotis coast) and that to the east of Port-Said (Sinai coast). The rate of Deltaic growth varies from place to place along the Mediterranean coast. This is evidenced by three headlands penetrating into the sea, one at Ras El-Barr (Damietta), another at Baltim (Kafr El-Sheikh) and the third at Rosetta (El- Behira). The soils of the Nile Delta are heavy in texture, rather compact at the surface and rich in humus (El-Gabaly et al., 1969a). According to the map of the world distribution of the arid regions 
(UNESCO, 1977), the soils of the study area are man variants of Gleysoils and Fluvisoils that belong to the Pliocene and Pleistocene (El-Gabaly et al., 1969b). Deposits covering the Delta reach about $10.9 \mathrm{~m}$ in thickness. These deposits are composed mainly of silt, clay, sandy clay with biotite, magnetite and limestone formations, these deposites are considered as the basis of Egypt's fertility (Abu Al-Izz, 1971). Ayyad and ElGharbeeb (1984) mentioned that the Mediterranean coastal region of Egypt belongs to the dry arid climate zone of Koppen's classification system (1931), the arid mesothermal province of Thornthwaite (1948), and the Mediterranean coastal arid bioclimatic zone of Emberger (1955). The mean maximum air temperature ranged between $18.1{ }^{\circ} \mathrm{C}$ in January at Rosetta to $34{ }^{\circ} \mathrm{C}$ in July at Mansoura, and the mean minimum air temperature varied from $6.8{ }^{\circ} \mathrm{C}$ in January at Mansoura to $24.9{ }^{\circ} \mathrm{C}$ in August at Rosetta. The small amount of rainfall $(100-150 \mathrm{~mm})$ in the study area falls during winter season (November-March), while summer is practically dry and hot. The relative humidity varied from $69 \%$ in summer to $84 \%$ in winter.

The Mediterranean coastal region of Egypt can be divided into three sections: 1) the western section (Mareotis coast) between Abu Qir at east to Sallum at west for about $550 \mathrm{~km}$ long, 2) the middle section (Deltaic coast) between Port- Said at east to Abu Qir at west for about $220 \mathrm{~km}$ long and 3) the eastern section (Sinai coast) between Rafah at east to Port-Said at west for about $200 \mathrm{~km}$ long. The vegetation of the coastal zone is considered the richest one among the phytogeographical regions of Egypt, where its flora represents more than $50 \%$ of the total flora of Egypt (Hassib,1951; Täckholm,1974).

The Deltaic Mediterranean coast can be distinguished into four main habitats:1) Sand formations (mounds, sheets and sand dunes), 2) Salt marshes (black barren, wet and dry), 3) Fertile sandy lands (cultivated and non-cultivated) and 4) Reed swamps. The sand formations are basically siliceous deposits mixed with clay and silt particles. The sand dunes are considered as the main geomorphic features especially in western part of this coast. These dunes can be classified into three types: mobile dunes, partial stabilized dunes and stabilized dunes (Mashaly, 2001\&2002; Mashaly et al., 2008). Floristically, Poaceae is considered as the richest family in the Deltaic Mediterranean coast, where it dominates all habitats of this area, especially the sand formation habitat type.

Grasses (constitute the largest and most valuable family among the flowering plants, with about 10000 species in the flora of Egypt, it includes 277 species (Boulos, 2009). Grasses occupy wide tracts of land and they are evenly distributed in all parts of the world. They occur in every soil, in all kinds of situation and under all climatic conditions. Although several families of plants supply the wants of man, the grass family exceeds all the others in the amount and the value of its products. The grasses growing in pasture land and the cereals grown all over the world are of more value to man and his domestic animals than all the other plants taken together (Rangachari and Tadulingam, 2007).
Therefore, the selection of some natural grasses in the present study is required and urgent. Therefore, the main objective of the present investigation aims to provide insight on the floristic compition and vegetation analysis of flora associated with some grasses growing naturally in sand formations in the Delta Nile Coast of Egypt.

\section{The Study Area}

The sampled stands were distributed in the sand formations in the Deltaic Mediterranean coast of Egypt (Figure1). The geologic and vegetation structures of the Nile Delta as a part of the northern Egypt has been subjected to the same geologic events that affected the whole region during its Pre-Miocene geological history. The major elements of geomorphology of Deltaic section were given by (Sestini, 1976). These elements comprise two main geomorphological components: coastal and deltaic. (Mashaly, 1987) stated that the Deltaic coastal section is characterized by morphological structure basically different from that to the west of Abu-Qir Bay (Mareotis coast) and that to the east of Port-Said (Sinai coast). The rate of Deltaic growth varies from place to place along the Mediterranean coast. This is evidenced by three headlands penetrating into the sea, one at Ras El-Barr (Damietta), another at Baltim (Kafr El-Sheikh) and the third at Rosetta (El- Behira). The soils of the Nile Delta are heavy in texture, rather compact at the surface and rich in humus (El-Gabaly et al., 1969a). According to the map of the world distribution of the arid regions (UNESCO, 1977), the soils of the study area are man variants of Gleysoils and Fluvisoils that belong to the Pliocene and Pleistocene (El-Gabaly et al., 1969b). Deposits covering the Delta reach about $10.9 \mathrm{~m}$ in thickness. These deposits are composed mainly of silt, clay, sandy clay with biotite, magnetite and limestone formations, these deposites are considered as the basis of Egypt's fertility (Abu Al-Izz, 1971). Ayyad and ElGharbeeb (1984) mentioned that the Mediterranean coastal region of Egypt belongs to the dry arid climate zone of Koppen's classification system (1931), the arid mesothermal province of Thornthwaite (1948), and the Mediterranean coastal arid bioclimatic zone of (Emberger, 1955). The mean maximum air temperature ranged between $18.1^{\circ} \mathrm{C}$ in January at Rosetta to $34^{\circ} \mathrm{C}$ in July at Mansoura, and the mean minimum air temperature varied from $6.8^{\circ} \mathrm{C}$ in January at Mansoura to $24.9^{\circ} \mathrm{C}$ in August at Rosetta. The small amount of rainfall $(100-150 \mathrm{~mm})$ in the study area falls during winter season (November-March), while summer is practically dry and hot. The relative humidity varied from $69 \%$ in summer to $84 \%$ in winter.

\section{MATERIAL AND METHODS}

During spring 2016, number of stands (36) was surveyed to represent five selected annual and perennial grasses namely. Aegilops bicornis (Forssk.) Jaub \& Spach, Bromus diandrus Roth, Elymus farctus (Viv.) Runem.ex Melderis, Hordeum murinum L.and Stipagrostis lanata (Forssk.) De Winter. Thirty six stands $(10 \mathrm{~m} \times 10 \mathrm{~m}$ each) were chosen for sampling 


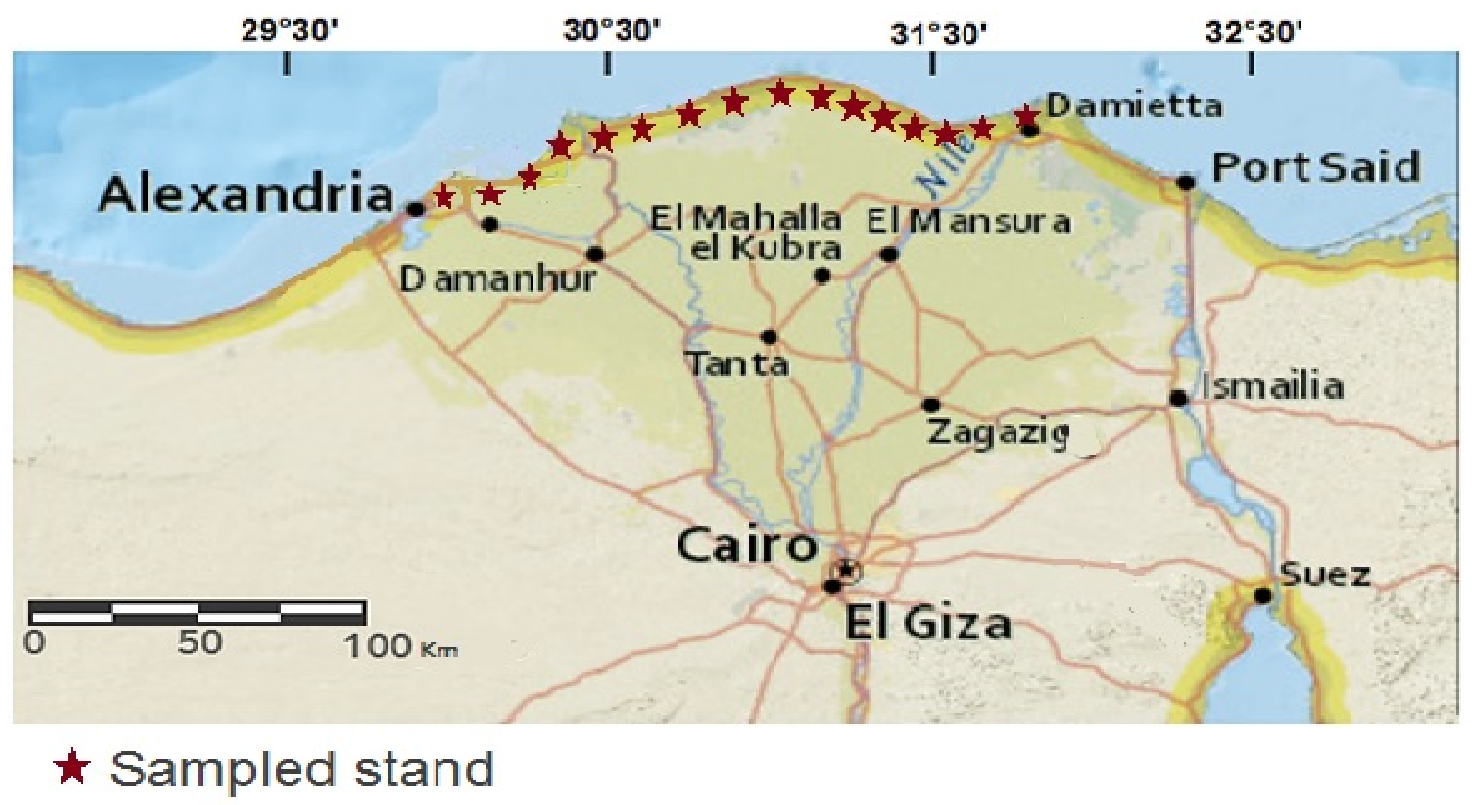

Figure (1). Map showing the location of the sampled stands in study area.

vegetation in the present study. These stands were selected to cover all physiographic variations to ensure sampling of wide range of vegetation diversity. The density and cover of each plant species were calculated in each stand. Density was obtained by counting the number of individuals of the species within a series of randomly distributed stands (Shukla and Candel, 1989). Cover was measured by application of line intercept method (Canfield, 1941). The importance value index (IV = out of 200) was calculated by the summation of relative values of density and cover for each species (Muller-Dombois and Ellenberg, 1974). From each sand three soil samples were collected from depth $0-50 \mathrm{~cm}$, pooled together to from one composite sample, spread over sheets of paper, air dried, passed through $2 \mathrm{~mm}$ sieve, and packed in plastic bags ready for physical and chemical analyses. For the determination of particle sizes of soil samples (soil texture), drying sieves method (mechanical analysis) and the percentages of sand, silt and clay were calculated (Piper, 1947). Hilgard Pan Box was used for the estimation of maximum water holding capacity of the soil samples (Piper, 1947). The volumes of pores occupied by water and pores occupied by air (soil porosity) were determined by measuring flask method as described by Piper, 1947.The calcium carbonate was dissolved in excess of $\mathrm{HCl}(1 \mathrm{~N})$ and then determined by titration against $1 \mathrm{~N} \mathrm{NaOH}$ and expressed as percentage (Jackson, 1967), organic carbon was determined using Walkely and Black rapid titration method (Piper, 1947). Electrical-pH meter (Model Corning, NY 14831 USA) digital analyzer with glass electrode was used to determine the soil reaction of soil samples (Jackson, 1967) and electrical conductivity was measured by conductivity meter (Model Corning, NY 14831 USA) and expressed as mmhos/cm (Jackson, 1967). Chlorides in the soil extract were determined by titration with $\mathrm{N} / 35.5$ $\mathrm{AgNO}_{3}$ in presence of $\mathrm{K}_{2} \mathrm{CrO}_{4}$ as an indicator (Jackson,
1967), sulphates were estimated gravimetrically using $\mathrm{BaCl}_{2}$ solution. The sulphates were precipitated by barium chloride solution as barium sulphate and ignited in muffle furnace at $700-800{ }^{\circ} \mathrm{C}$ (Piper, 1947). Bicarbonates were determined by titration method using $0.1 \mathrm{~N} \mathrm{HCl}$ using methyl orange as indicator (Pierce et al., 1958). Total soluble phosphorus was determined by digestion and followed by direct stannous chloride method as described in American Public Health Association (APHA, 1998). The total nitrogen was determined by the conventional semimicromodification of Kjeldahl method (Pirie, 1955). $\mathrm{Na}$ and $\mathrm{K}$ cations were determined in soil samples by Flame Photometer (Model PHF $80 \quad \mathrm{P}$ Biologie Spectrophotometer), while $\mathrm{Ca}, \mathrm{Mg}, \mathrm{Fe}, \mathrm{Mn}, \mathrm{Zn}, \mathrm{Cu}$, $\mathrm{Co}, \mathrm{Cd}$ and $\mathrm{Pb}$ were estimated using Atomic Absorption Spectrometer (A Perkin-Elmer, Model 2380.USA) (Allen et al., 1974).Two multivariate analysis techniques (classification and ordination) were applied. The classification technique applied here was the two way indicator species analysis (TWINSPAN) using the CAP software for windows version 1.2 (Henderson and Seaby,1999) The performed ordination techniques were the Detrended Correspondence Analysis (DCA) and the Canonical Correspondence Analysis (CCA) using computer program MVSP, version 3.1 (Ter Braak, 2003). The identification, nomenclature and chorotypes of the recorded plant species were according to (Täckholm, 1974), (Zahary, 1966 \& 1972); Feinbrun-Dothen (1978 \&1986) and (Boulos, 1999-2005 \& 2009).

\section{RESULTS}

\section{Floristic Features}

The floristic features of Deltaic Mediterranean coast of Egypt in the present study is listed in Table (1). In total, 79 plant species belonging to 70 genera and 26 families were recorded in 36 sampled stands on the 
sand formations in the Deltaic Mediterranean coast of Egypt. Asteraceae included 15 species, followed by Poaceae (12 species), then Chenopodiaceae (7 species). Brassicaceae and Fabaceae (5 species each) and Polygonaceae (4 species). These six families were the species rich families where they constituted the main bulk of the flora in the present study (48 species $=$ $60.76 \%$ ).

\section{Life span}

The life-span of the recorded species (79) includes 44 annuals (56.69\%) and 33 perennials (44.30\%).

\section{Life form}

The life-form spectra of the recorded species include 44 species as therophytes $(55.69 \%)$, followed by 13 species as cryptophytes $(16.45 \%)$, then eight species hemicryptophytes $(10.12 \%)$ and seven species as chamaephytes and phanerphytes for each $(8.86 \%)$.

\section{Chorological analysis}

The chorological affinity was presented in Table (2). The Saharo-Sindian element was represented by 34 species which can be distinguished into 15 Biregional, 11Pluriregional and eight Monoregional elements. Irano-Turanian element was represented by 21 species which can be also distinguished into 11 Pluriregional and ten Biregional elements . Euro-Siberian element was represented by ten species which may be distinguished into eight Pluriregional and two Biregional elements. Sudano-Zambezian element was represented by nine species which distinguished into seven Biregional and two Pluriregional elements. Cosmopoliton element was represented by six species, three species as Pantropical and one species for each Palaetropical ,Neotropical and Cultivated and Naturalized elements. In general as shown in Table (2), the Biregional elements were represented by 29 species (36.72\%), followed by the Monoregional elements 22 species $(27.85 \%)$, then the Pluriregional elements 16 species $(20.25 \%)$, and the world wide elements by 11 species $(13.92 \%)$.

\section{Vegetation Structure \\ Classification of sampled stands}

The application of TWINSPAN classification based on the importance values ( out of 200 ) of 79 plant species recorded in 36 sampled stands in the present study, led to the identification of four vegetation groups (A, B, C and D; Figure 2). The vegetation composition of these groups was presented in Table (3). The vegetation groups were named according to the 1 st and 2nd dominant species with the highest importance value in each group.

Vegetation group A included 21 species distributed in 4 stands. The codominant species were Elymus farctus $(\mathrm{IV}=35.83)$ and Calligonum polygonoides $(\mathrm{IV}=31.84)$. The most important associated species in this group include Zygophyllum aegyptium (IV=21.29) and Rumex pictus (IV=16.22), but the indicator species has not been detected. Group B comprised 13 stands with 50 species.
Hordeum murinum (IV=20.95) was the dominant species. Bromus diandrus $(\mathrm{IV}=10.73)$ and Senecio glaucus (IV=10.47) were recorded as the most important associated species in this community. The indicator species of this group included Lactuca serriola (IV=1.90), Tamarix tetragyna ( $\mathrm{IV}=6.86)$, Lotus halophilus $(\mathrm{IV}=1.30), \quad$ Melilotus indicus $(\mathrm{IV}=2.75)$ and Rumex pictus $(\mathrm{IV}=6.26)$.

Group C comprised 11 stands with 38 species, and it was codominated by Stipagrostis lanata $(\mathrm{IV}=24.83)$ and Echinops spinosus (IV=22.85). Rumex pictus $(\mathrm{IV}=15.33)$ and Moltkiopsis ciliata $(\mathrm{IV}=12.79)$ were the most important associated species in this community. The indicator species in this group comprise Plantago squarrosa $(\mathrm{IV}=7.33)$ and Senecio glaucus (IV=7.39).

Group D included 43 species in 8 stands, and it was codominated by Lycium schweinfurthii $(\mathrm{IV}=25.03)$ and Echinops spinosus $(\mathrm{IV}=23.80)$. The indicator species in this group comprise Atriplex halimus $(\mathrm{IV}=2.13)$ and Rumex pictus $(\mathrm{IV}=5.16)$. The most important associated species in this community included Stipagrostis lanata (IV=11.61), Erodium laciniatum $(\mathrm{IV}=11.44)$ and Moltkiopsis ciliata $(\mathrm{IV}=10.80)$.

\section{Ordination of sampled stands}

The Detrended Correspondence Analysis (DCA) ordination plot of the 36 sampled stands on axes 1 and 2 is shown in Figure (3). Group A codominted by Elymus farctus and Calligonum polygonoides was separated at the uppermost part of DCA diagram. On the other hand, group $\mathrm{B}$ dominated by Hordeum murinum was segregated at the lower part of DCA diagram. Both groups A and B were superimposed. Group C codominated by Stipagrostis lanata and Echinops spinosus was segregated at lower part of the left side of the DCA diagram, while group D codominated by Lycium schweinfurthii and Echinops spinosus was separated at the lower part of the right side of the DCA. It is clear that the identified four vegetation groups (A-D) were obviously superimposed with unclear pattern of segregation on the ordination (DCA) plane.

\section{Vegetation- Soil Relationships \\ Relationship between soil parameters and vegetation groups}

The variation of soil variables between four vegetation groups recognized by the TWINSPAN classification was shown in Table (4).

The highest percentage of sand was attained in group (D), while the lowest percentage was estimated in group (C). The highest percentage of silt fraction was attained in group (C), while the lowest percentage was determined in group (D). The highest percentage of clay fraction was attained in group (B), while the lowest percentage was estimated in group (D). Soil porosity attained the highest value in group (C), while the lowest value in group (A). Maximum water holding capacity showed the highest value in group (B), while it showed the lowest value in group (D).

The chemical soil variables showed variations from 


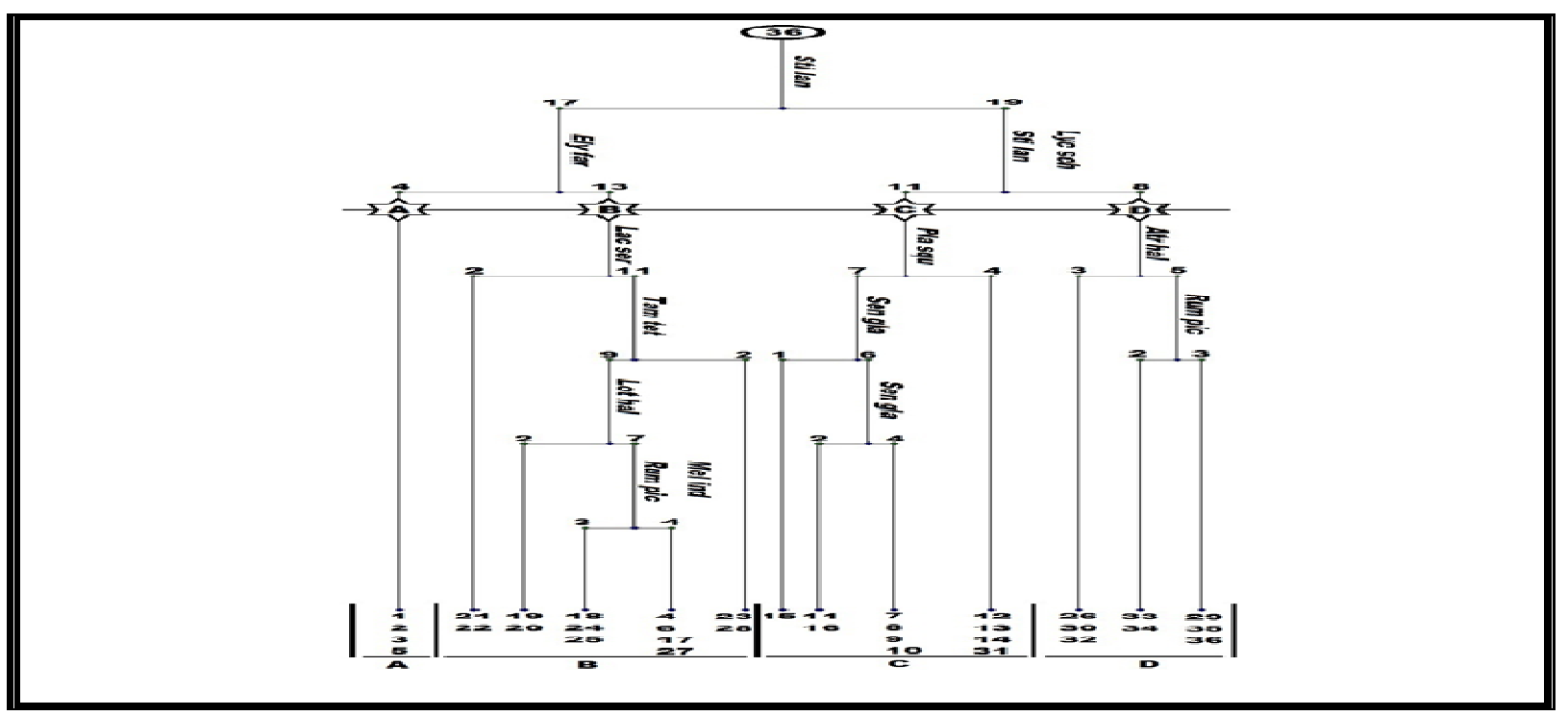

Figure (2):TWINSPAN dendrogram recognizing four groups (A, B, C and D) at the third level. The indicator species are abbreviated by the first three letters of genus and species, respectively. The indicator species are coded as follow: Sti lan = Stipagrostis lanata; Ely far = Elymus farctus; Lyc sch = Lycium schweinfurthii; Lac ser = Lactuca serriola; Pla squ = Plantago squarraso; Atr hal $=$ Atriplex halimus; Tam tet $=$ Tamarix tetragyna; Sen gla = Senecio glaucus; Rum pic = Rumex pictus; Lot hal = Lotus halophilus; Mel ind = Melilotus indicus.

Table (1): Species composition of the selected grass species in the study area, with their families, life span, life-forms and chorotypes. Ann: Annuals; Per: Perennials; Th: Therophytes; Ch: Chamaephytes; H: Hemicryptophytes; Ph: Phanerophytes; Cr: Cryptophytes; ME: Mediterranean; SA-SI: Saharo-Sindian; IR-TR: Irano-T uranian; S-Z: Sudano-Zambezian; ER-SR: Euro-Siberian; PAL: Palaeotropical, COSM: Cosmopolitan; PAN: Pantropical; NEO: Neotropical; , CULT and NAT: Cultivated and Naturalized.

\begin{tabular}{|c|c|c|c|}
\hline Family and Species & $\begin{array}{l}\text { Life } \\
\text { span }\end{array}$ & Life form & Chorotype \\
\hline \multicolumn{4}{|l|}{ Aizocaceae } \\
\hline Mesembryanthemum crystallinum $\mathrm{L}$. & Ann. & Th & ME+ER-SR \\
\hline \multirow{2}{*}{\multicolumn{4}{|c|}{$\begin{array}{l}\text { M. noafflorum L. } \\
\text { Amaryllidaceae }\end{array}$}} \\
\hline & & & \\
\hline Pancratium maritimum $\mathrm{L}$. & Per. & $\mathrm{Cr}$ & ME \\
\hline \multicolumn{4}{|l|}{ Apiaceae } \\
\hline Bupleurum semicompositum $\mathrm{L}$. & Ann. & Th & $\mathrm{ME}+\mathrm{IR}-\mathrm{TR}+\mathrm{SA}-\mathrm{SI}$ \\
\hline Daucus litoralis Sm. & Ann. & Th & $\mathrm{ME}$ \\
\hline Pseudorlaya pumila (L.) Grande & Ann. & Th & ME \\
\hline \multicolumn{4}{|l|}{ Asclepiadaceae } \\
\hline Cynanchum acutum $\mathrm{L}$. & Per. & $\mathrm{H}$ & ME+IR-TR \\
\hline \multicolumn{4}{|l|}{ Asparagaceae } \\
\hline \multirow{2}{*}{\multicolumn{4}{|c|}{$\begin{array}{l}\text { Asparagus stipularts ForssK. } \\
\text { Asteraceae }\end{array}$}} \\
\hline & & & \\
\hline Atractylis carduus (Forssk.) C.Chr. & Per. & $\mathrm{H}$ & $\mathrm{ME}+\mathrm{SA}-\mathrm{SI}$ \\
\hline Carduus getulus Pomel & Ann. & Th & SA-SI \\
\hline C. pycnocephalus L. & Ann. & Th & $\mathrm{ME}+\mathrm{IR}-\mathrm{TR}$ \\
\hline Carthamus tenuis (Boiss \& Blanche) Bornm. & Ann. & Th & ME \\
\hline Centaurea glomerata Vahl & Ann. & Th & ME \\
\hline Echinops spinosus L. & Per. & $\mathrm{H}$ & $\mathrm{ME}+\mathrm{SA}-\mathrm{SI}$ \\
\hline Ifloga spicata (Forssk.) Sch.Bip. & Ann. & Th & SA-SI \\
\hline Lactuca serriola $\mathrm{L}$. & Ann. & Th & $\mathrm{ME}+\mathrm{IR}-\mathrm{TR}+\mathrm{ER}-\mathrm{SR}$ \\
\hline Launaea mucronata (Forssk.) Muschl. & Per. & $\mathrm{H}$ & ME+SA-SI \\
\hline Limbardia crithmoides (L.) Dumort. & Per. & $\mathrm{Ch}$ & $\mathrm{ME}+\mathrm{ER}-\mathrm{SR}+\mathrm{SA}-\mathrm{SI}$ \\
\hline Reichardia tingitana (L.) Roth & Ann. & $\mathrm{Th}$ & ME+SA-SI+IR-TR \\
\hline Senecio glaucus L. & Ann. & Th & $\mathrm{ME}+\mathrm{SA}-\mathrm{SI}+\mathrm{IR}-\mathrm{TR}$ \\
\hline Sonchus bulbous (L.) N.Kilian \&Greuter & Per. & $\mathrm{Cr}$ & $\mathrm{ME}$ \\
\hline S. oleraceus L. & Ann. & Th & COSM \\
\hline Urospermum picroides (L.) F.W. Schmidt & Ann. & $\mathrm{Th}$ & ME+IR-TR \\
\hline \multicolumn{4}{|l|}{ Boraginaceae } \\
\hline Anchusa humilis (Desf.) I.M. Johnst. & Ann. & Th & $\mathrm{ME}+\mathrm{SA}-\mathrm{SI}$ \\
\hline Heliotropium curassavicum L. & Per. & $\mathrm{Ch}$ & NEO \\
\hline \multirow{2}{*}{\multicolumn{4}{|c|}{ Brassicaceae }} \\
\hline & & & \\
\hline Brassica tournefortii Gouan & Ann. & Th & ME+IR-TR+SA-SI \\
\hline Cakile maritima Scop. subsp. aegyptiaca (Willd.) Nyman & Ann. & Th & ME+ER-SR \\
\hline
\end{tabular}


Table (1) continue

\begin{tabular}{|c|c|c|c|}
\hline Family and Species & Life span & Life form & Chorotype \\
\hline Lobularia arabica (Boiss.) Muschl. & Ann. & $\mathrm{Th}$ & SA-SI \\
\hline L. libyca (Viv.) C.F.W.Meissn. & Ann. & $\mathrm{Th}$ & SA-SI \\
\hline Maresia pygmaea (DC.) O.E.Schulz & Ann. & $\mathrm{Th}$ & SA-SI \\
\hline \multicolumn{4}{|l|}{ Caryophyllaceae } \\
\hline Paronychia arabica (L.) DC. & Ann. & $\mathrm{Th}$ & $\mathrm{SA}-\mathrm{SI}+\mathrm{ME}+\mathrm{S}-\mathrm{Z}$ \\
\hline Silene succulenta Forssk. & Per. & $\mathrm{H}$ & ME \\
\hline S. viviani Steud. & Ann. & $\mathrm{Th}$ & $\mathrm{ME}+\mathrm{SA}-\mathrm{SI}$ \\
\hline \multicolumn{4}{|l|}{ Chenopodiaceae } \\
\hline Atriplex halimus L. & Per. & $\mathrm{Ph}$ & $\mathrm{ME}+\mathrm{SA}-\mathrm{SI}$ \\
\hline Bassia indica (Wight) A.J.Scott & Ann. & $\mathrm{Th}$ & S-Z+IR-TR \\
\hline Chenopodium album $\mathrm{L}$. & Ann. & $\mathrm{Th}$ & COSM \\
\hline C. murale $\mathrm{L}$. & Ann. & $\mathrm{Th}$ & COSM \\
\hline Halocnemum strobilaceum (Pall.) M.Bieb. & Per. & $\mathrm{Ch}$ & SA-SI \\
\hline Salsola kali L. & Ann. & $\mathrm{Th}$ & COSM \\
\hline Suaeda vera Forssk.ex.J.F.Gmel. & Per. & $\mathrm{Ch}$ & $\mathrm{ME}+\mathrm{SA}-\mathrm{SI}+\mathrm{ER}-\mathrm{SR}$ \\
\hline \multicolumn{4}{|l|}{ Convolvulaceae } \\
\hline Cressa cretica L. & Per. & $\mathrm{H}$ & $\mathrm{ME}+\mathrm{PAL}$ \\
\hline \multicolumn{4}{|l|}{ Cyperaceae } \\
\hline Cyperus capitatus Vand. & Per. & $\mathrm{Cr}$ & $\mathrm{ME}$ \\
\hline C. conglomeratus Rottb. & Per. & $\mathrm{Cr}$ & $\mathrm{SA}-\mathrm{SI}+\mathrm{S}-\mathrm{Z}$ \\
\hline C. rotundus $\mathrm{L}$. & Per. & $\mathrm{Cr}$ & PAN \\
\hline \multicolumn{4}{|l|}{ Euphorbiacea } \\
\hline Ricinus communis L. & Per. & $\mathrm{Ph}$ & CULT and NAT \\
\hline \multicolumn{4}{|l|}{ Fabaceae } \\
\hline Alhagi graecorum Boiss. & Per. & $\mathrm{H}$ & PAL \\
\hline Lotus halophilus Boiss. \& Spruner & Ann. & $\mathrm{Th}$ & $\mathrm{ME}+\mathrm{SA}-\mathrm{SI}$ \\
\hline Melilotus indicus (L.) All. & Ann. & $\mathrm{Th}$ & ME+IR-TR+SA-SI \\
\hline Ononis serrata Forssk. & Ann. & $\mathrm{Th}$ & $\mathrm{ME}+\mathrm{SA}-\mathrm{SI}$ \\
\hline $\begin{array}{l}\text { Retama raetam (Forssk.) Webb \& Berthel. } \\
\text { Geraniaceae }\end{array}$ & Per. & $\mathrm{Ph}$ & SA-SI+IR-TR \\
\hline $\begin{array}{l}\text { Erodium laciniatum (Cav.) Willd. } \\
\text { Juncaceae }\end{array}$ & Ann. & $\mathrm{Th}$ & $\mathrm{ME}$ \\
\hline $\begin{array}{l}\text { Juncus acutus L. } \\
\text { Malvaceae }\end{array}$ & \multicolumn{2}{|c|}{ Malvaceae } & $\mathrm{ME}+\mathrm{IR}-\mathrm{TR}+\mathrm{ER}-\mathrm{SR}$ \\
\hline \multicolumn{4}{|l|}{ Neuradaceae } \\
\hline Neurada procumbens L. & Ann. & $\mathrm{Th}$ & SA-SI+IR-TR \\
\hline \multicolumn{4}{|l|}{ Plantaginaceae } \\
\hline Plantago squarrosa Murray & Ann. & $\mathrm{Th}$ & $\mathrm{ME}$ \\
\hline \multicolumn{4}{|l|}{ Plumbaginaceae } \\
\hline $\begin{array}{l}\text { Limoniastrum monopetalum (L.) Boiss. } \\
\text { Poaceae }\end{array}$ & Per. & $\mathrm{Ch}$ & SA-SI \\
\hline Aegilops bicornis (Forssk.) Jaub \& Spach & Ann. & $\mathrm{Th}$ & $\mathrm{ME}+\mathrm{SA}-\mathrm{SI}$ \\
\hline Cutandia memphitica (Spreng.) Benth. & Ann. & Th & $\mathrm{ME}+\mathrm{IR}-\mathrm{TR}+\mathrm{SA}-\mathrm{SI}$ \\
\hline Cynodon dactylon (L.) Pers. & Per. & $\mathrm{Cr}$ & PAN \\
\hline Elymus farctus (Viv.) Runem.ex Melderis & Per. & $\mathrm{Cr}$ & ME \\
\hline Hordeum murinum $\mathrm{L}$. & Ann. & Th & ME+IR-TR+ER-SR \\
\hline Imperata cylindrica (L.) Raeusch. & Per. & $\mathrm{H}$ & $\mathrm{PAL}+\mathrm{ME}$ \\
\hline Lolium multiflorum Lam. & Ann. & Th & $\mathrm{ME}+\mathrm{IR}-\mathrm{TR}+\mathrm{ER}-\mathrm{SR}$ \\
\hline Parapholis incurva (L.) C.E.Hubb & Ann. & Th & $\mathrm{ME}+\mathrm{IR}-\mathrm{TR}+\mathrm{ER}-\mathrm{SR}$ \\
\hline Phalaris minor Retz. & Ann. & Th & ME+IR-TR \\
\hline Phragmites australis (Cav.) Trin. ex Steud. & Per. & $\mathrm{Cr}$ & COSM \\
\hline Stipagrostis lanata (Forssk.) De Winter & Per. & $\mathrm{Cr}$ & SA-SI \\
\hline \multicolumn{4}{|l|}{$\begin{array}{l}\text { Polygonaceae } \\
\text { Pon }\end{array}$} \\
\hline $\begin{array}{l}\text { Calligonum polygonoides L. subsp. comosum (L' Her.) } \\
\text { Soskov }\end{array}$ & Per. & $\mathrm{Ph}$ & SA-SI+IR-TR \\
\hline Emex spinosa (L.) Campd. & Ann. & Th & $\mathrm{ME}+\mathrm{SA}-\mathrm{SI}$ \\
\hline Polygnum equisetiforme $\mathrm{Sm}$. & Per. & $\mathrm{Cr}$ & ME+IR-TR \\
\hline \multirow{2}{*}{\multicolumn{4}{|c|}{ Solanaceae }} \\
\hline & & & \\
\hline Solanum nigrum $\mathrm{L}$. & Ann. & Th & COSM \\
\hline \multirow{2}{*}{\multicolumn{4}{|c|}{ Tamaricaceae }} \\
\hline & & & \\
\hline Tamarix nilotica (Ehrenb). Bunge & Per. & $\mathrm{Ph}$ & SA-SI+S-Z \\
\hline T. tetragyna Ehrenb. & Per. & $\mathrm{Ph}$ & $\mathrm{SA}-\mathrm{SI}+\mathrm{S}-\mathrm{Z}$ \\
\hline
\end{tabular}




\begin{tabular}{|c|c|c|c|}
\hline Family and Species & Life span & Life form & Chorotype \\
\hline $\begin{array}{l}\text { Typhaceae } \\
\text { Typha domingensis (Pers.) Poir.ex Steud. } \\
\text { Zygophyllaceae }\end{array}$ & Per. & $\mathrm{Cr}$ & PAN \\
\hline Zygophyllum aegyptium Hosny & Per. & $\mathrm{Ch}$ & $\mathrm{ME}$ \\
\hline
\end{tabular}

Table (2): Summarized chorological analysis of the recorded flora in the study area.

\begin{tabular}{|c|c|c|c|}
\hline Chorotype & No & $\%$ & Geographical distribution \\
\hline ME & 14 & 17.72 & \\
\hline SA-SI & 8 & 10.126 & Mono-regional elements \\
\hline Sub Total & 22 & 27.85 & \\
\hline $\mathrm{ME}+\mathrm{SA}-\mathrm{SI}$ & 12 & 15.189 & \\
\hline ME+IR-TR & 6 & 7.59 & \\
\hline IR-TR+S-Z & 4 & 5.06 & \\
\hline SA-SI+S-Z & 3 & 3.79 & Bi-regional elements \\
\hline $\mathrm{ME}+\mathrm{ER}-\mathrm{SR}$ & 2 & 2.53 & \\
\hline $\mathrm{ME}+\mathrm{PAL}$ & 2 & 2.53 & \\
\hline Sub Total & 29 & 36.72 & \\
\hline ME+IR-TR+SA-SI & 6 & 7.59 & \\
\hline ME+IR-TR+ER-SR & 5 & 6.32 & \\
\hline $\mathrm{ME}+\mathrm{ER}-\mathrm{SR}+\mathrm{SA}-\mathrm{SI}$ & 3 & 3.79 & Pluri-regional elements \\
\hline $\mathrm{ME}+\mathrm{SA}-\mathrm{SI}+\mathrm{S}-\mathrm{Z}$ & 2 & 2.53 & \\
\hline Sub Total & 16 & 20.25 & \\
\hline COSM & 6 & 7.59 & \\
\hline PAN & 3 & 3.79 & \\
\hline PAL & 1 & 1.26 & World-wide elements \\
\hline NEO & 1 & 1.26 & \\
\hline Sub Total & 11 & 13.92 & \\
\hline CULT \& NAT & 1 & 1.26 & CULT \&NAT \\
\hline Total & 79 & 100 & \\
\hline
\end{tabular}


Ecological Study on some Grasses Growing Naturally

Table (3) Mean importance values (out of 200) and coefficient of variation (between brackets) of recorded species in the different vegetation groups resulting from the TWINSPAN. classification.

\begin{tabular}{|c|c|c|c|c|c|}
\hline No. & Species & Group A & Group B & Group C & Group D \\
\hline & Total number of stands & 4 & 13 & 11 & 8 \\
\hline 1 & Aegilops bicornis & - & $9.38(0.093)$ & $0.84(3.31)$ & $4.30(1.58)$ \\
\hline 2 & Alhagi graecorum & - & $0.85(3.60)$ & $5.125(1.76)$ & - \\
\hline 3 & Anchusa humilis & - & $4.63(1.69)$ & - & $2.13(1.39)$ \\
\hline 4 & Asparagus stipularis & - & - & $0.95(3.31)$ & $7.80(0.65)$ \\
\hline 5 & Atractylis carduus & $3.26(2)$ & - & $0.36(3.31)$ & $2.33(1.38)$ \\
\hline 6 & Atriplex halimus & - & $0.66(3.60)$ & - & $14.92(0.88)$ \\
\hline 7 & Bassia indica & - & $0.57(3.60)$ & - & - \\
\hline 8 & Brassica tourmefortii & $3.70(2)$ & $7.31(1.46)$ & $1.33(3.317)$ & $1.63(1.89)$ \\
\hline 9 & Bromus diandrus & $5.87(2)$ & $10.73(1.257)$ & $1.23(3.31)$ & $0.55(2.82)$ \\
\hline 10 & Bupleurum semicompositum & - & - & - & $0.99(2.82)$ \\
\hline 11 & Cakile maritima & $15.24(0.533)$ & $9.01(0.707)$ & $2.66(2.28)$ & $0.43(2.82)$ \\
\hline 12 & Calligonum polygonoides & $31.84(0.852)$ & - & - & $4.43(2.19)$ \\
\hline 13 & Carduus getulus & - & - & $0.63(3.31)$ & $3.60(1.13)$ \\
\hline 14 & Carduus pycnocephalus & - & $4.13(2.126)$ & - & $0.78(2.82)$ \\
\hline 15 & Carthames tenuis & $2.09(2)$ & $5.75(1.64)$ & - & $2.80(1.49)$ \\
\hline 16 & Centaurea glomerata & - & $0.86(3.60)$ & - & $9.05(0.64)$ \\
\hline 17 & Chenopodium album & - & $0.77(2.44)$ & - & - \\
\hline 18 & Chenopodium murale & - & $6.06(0.933)$ & - & - \\
\hline 19 & Cressa cretica & - & - & - & $0.78(2.82)$ \\
\hline 20 & Cutandia memphitica & $8.89(1.30)$ & - & $0.38(3.31)$ & - \\
\hline 21 & Cynanchum acutum & - & $0.616(3.60)$ & - & $0.65(2.82)$ \\
\hline 22 & Cynodon dactylon & - & $1.44(2.63)$ & - & $1.57(1.88)$ \\
\hline 23 & Cyperus capitatus & - & - & $3.73(1.95)$ & $3.54(1.12)$ \\
\hline 24 & Cypers conglomeratus & - & $0.19(3.60)$ & $0.84(3.31)$ & $8.57(0.52)$ \\
\hline 25 & Daucus litoralis & - & - & $7.22(0.99)$ & $3.44(1.12)$ \\
\hline 26 & Echinops spinosus & - & $2.55(2.81)$ & $22.85(0.70)$ & $23.8(0.738)$ \\
\hline 27 & Elymus farctus & $35.83(0.456)$ & - & $8.48(1.58)$ & - \\
\hline 28 & Emex spinosa & - & $3.18(1.61)$ & - & $1.13(2.82)$ \\
\hline 29 & Erodium laciniatum & $1.99(2)$ & $5.92(1.174)$ & $8.58(1.012)$ & $11.44(0.414)$ \\
\hline 30 & Halocnemum strobilaceum & $10.12(2)$ & - & - & - \\
\hline 31 & Heliotropium curassavicum & - & $1.85(2.45)$ & - & - \\
\hline 32 & Hordeum marinum & $2.42(2)$ & $20.95(0.488)$ & $3.79(2.27)$ & - \\
\hline 34 & Ifloga spicata & $4.08(2)$ & - & $2.58(1.43)$ & - \\
\hline 35 & Imperata cylindrica & - & - & $7.58(1.21)$ & - \\
\hline
\end{tabular}


Mashaly et al.

\begin{tabular}{|c|c|c|c|c|c|}
\hline No. & Species & Group A & Group B & Group C & Group D \\
\hline 36 & Juncus acutus & - & $1.35(3.60)$ & - & - \\
\hline 37 & Lactuca serriola & - & $1.90(1.81)$ & - & - \\
\hline 38 & Launaea mucronata & $5.18(1.258)$ & $5.24(1.73)$ & $4.12(1.55)$ & $2.53(1.43)$ \\
\hline 39 & Limbarda crithmoides & - & $4.15(2.5)$ & - & - \\
\hline 40 & Limoniastrum monopetalum & - & $2.83(3.60)$ & - & $1.84(2.82)$ \\
\hline 41 & Lobularia arabica & - & - & $0.62(3.31)$ & $0.74(1.88)$ \\
\hline 42 & Lobularia libyca & - & - & $0.26(3.31)$ & - \\
\hline 43 & Lolium multiflorum & - & $1.425(1.93)$ & - & - \\
\hline 44 & Lotus halophilus & - & $1.30(2.45)$ & $12(0.716)$ & $1.28(2.37)$ \\
\hline 45 & Lycium schweinfurthii & - & $2.77(3.60)$ & - & $25.03(0.62)$ \\
\hline 46 & Malva parviflora & - & $9.40(0.845)$ & - & - \\
\hline 47 & Maresia pygmaea & - & - & $0.45(3.31)$ & - \\
\hline 48 & Melilotus indicus & - & $2.75(1.62)$ & - & - \\
\hline 49 & Mesembryanthemum crystallinum & - & $9.67(0.76)$ & $0.87(3.31)$ & $2.61(1.38)$ \\
\hline 50 & Mesembryanthemum nodiflorum & - & $2.04(2.44)$ & - & - \\
\hline 51 & Neurada procumbens & - & - & $6.09(1.67)$ & $3.24(1.30)$ \\
\hline 52 & Ononis serrata & - & - & $6.05(1.24)$ & $1.70(1.85)$ \\
\hline 53 & Pancratium maritimum & - & $0.72(3.60)$ & $3.97(2.23)$ & - \\
\hline 54 & Parapholis incurva & - & $1.17(3.60)$ & - & - \\
\hline 55 & Paronchyia arabica & $0.91(2)$ & - & $0.61(3.31)$ & $2.21(2.13)$ \\
\hline 56 & Phargmites australis & - & $4.10(1.45)$ & - & - \\
\hline 57 & Phalaris minor & - & $0.91(3.60)$ & - & - \\
\hline 58 & Plantago squarrosa & - & - & $7.33(1.59)$ & $2.73(1.42)$ \\
\hline 59 & Polygnum equisetiforme & - & $3.38(1.99)$ & - & $0.45(2.82)$ \\
\hline 60 & Pseudorlaya pumila & - & - & - & $0.43(2.82)$ \\
\hline 61 & Raphanus raphanistrum & - & $1.14(3.60)$ & - & - \\
\hline 62 & Reichardia tingitana & $1.715(2)$ & $7.82(0.87)$ & $1.88(2.25)$ & $1.88(1.49)$ \\
\hline 63 & Retama reatam & - & - & $5.95(3.31)$ & $2.30(2.82)$ \\
\hline 64 & Ricinus communis & - & $1.71(3.60)$ & - & - \\
\hline 65 & Rumex pictus & $16.22(0.728)$ & $6.26(1.16)$ & $15.33(0.426)$ & $5.16(0.84)$ \\
\hline 66 & Salsola kali & $4.79(2)$ & $0.36(3.60)$ & - & - \\
\hline 67 & Senecio glaucus & $9.17(0.712)$ & $10.47(0.55)$ & $7.39(0.79)$ & $1.17(1.92)$ \\
\hline 68 & Silene succulenta & - & - & $4.42(1.71)$ & - \\
\hline
\end{tabular}




\begin{tabular}{|c|c|c|c|c|c|}
\hline No. & Species & Group A & Group B & Group C & Group D \\
\hline 69 & Silene vivianii & - & - & $1.13(2.48)$ & - \\
\hline 70 & Solanum nigrum & - & $0.59(3.60)$ & - & - \\
\hline 71 & Sonchus bulbusa & - & - & - & - \\
\hline 72 & Sonchus oleraceus & - & $2.12(2.04)$ & - & - \\
\hline 73 & Stipagrostis lanata & $4.45(2)$ & - & $24.83(0.212)$ & $11.61(0.271)$ \\
\hline 74 & Suaeda vera & & - & - & $5.09(1.13)$ \\
\hline 75 & Tamarix nilotica & $10.92(2)$ & - & - & $5.34(1.89)$ \\
\hline 76 & Tamarix tetragyna & - & $6.86(2.44)$ & - & - \\
\hline 77 & Typha domingensis & - & $1.22(3.60)$ & - & - \\
\hline 78 & Urospermum picroides & - & $2.96(2.44)$ & - & - \\
\hline 79 & Zygophyllum aegyptium & $21.29(0.955)$ & $4.89(2.52)$ & $2.03(3.31)$ & - \\
\hline
\end{tabular}

one group to another. The highest values of organic carbon and $\mathrm{pH}$ were attained in group (C), while the lowest value of these variables was estimated in group (D). The highest value of electrical conductivity was attained in group (C), while the lowest value was estimated in group (B). Calcium carbonate content recorded the highest value in group (A), and the lowest value in group (D). The highest values of chlorides and bicarbonates were recorded in group $(\mathrm{C})$, while the lowest values of these variables were estimated in group (A). Sulphates recorded the highest value in group (D), while the lowest value in group (B).The highest values of total dissolved phosphorus and total nitrogen were determined in group $(\mathrm{C})$, while the lowest values of these variables were estimated in group (A).

On the other hand, the highest concentrations of the extractable sodium and calcium were determined in group $(\mathrm{C})$, while the lowest values of these variables were estimated in group (A). Group (C) also attained the highest concentrations of potassium and magnesium, while the lowest values of these variables were estimated in group (B).

In addition, the highest concentrations of the micro elements such as ferrous and copper were determined in group (B), while the lowest values of these variables were estimated in group (A). Group (C) attained the highest concentrations of the manganese and zinc, while the lowest values of these variables were estimated in group (A). Group (B) attained the highest concentrations of the cobalt and cadmium, while the lowest values of these variables were estimated in group (D). Lead showed the highest concentration in group (B), and the lowest concentration in group (C).

\section{Correlation between soil variables and vegetation gradients}

The correlation between vegetation and soil characteristics is indicated on the ordination diagram produced by Canonical Correspondence Analysis (CCA) of the biplot of the recorded species and soil variables (Figure 4). It is obvious that sulphates, calcium, magnesium, potassium, cobalt, calcium carbonates, cadmium and copper were the most effective soil variables controlling the distribution and abundance of the vegetation groups in the study area.

The characteristics codominat and the most important species of vegetation group C namely, Stipagrostis lanata, Echinops spinosus and Moltkiopsis ciliata were separated at the middle left quarter of CCA, where this group was closely related with calcium,sulphates,potassium,magnesium manganese,zinc and $\mathrm{pH}$. Codominat and leading species of vegetation group D namely, Lycium schweinfurthii, Echinops spinosus, Elymus farctus, Atriplex halimus and Erodium laciniatum were segregated at the lower-left quarter of CCA-diagram, where this group was obviously related with zinc, sand particle and porosity. On the other hand, the dominant and the most important species in group A namely, Elymus farctus, Calligonum polygonoides and Zygophyllum aegyptium were separated at the upper-right quarter of CCA, where this group was correlated with calcium carbonates. On the other hand, the dominant and leading species in group B namely, Hordeum murinum, Bromus diandrus and Senecio glaucus were separated at the lower-right quarter of CCA, where this group was correlated with cobalt, cadmium, copper, clay particle, maximum water holding capacity , silt fraction and total nitrogen. 


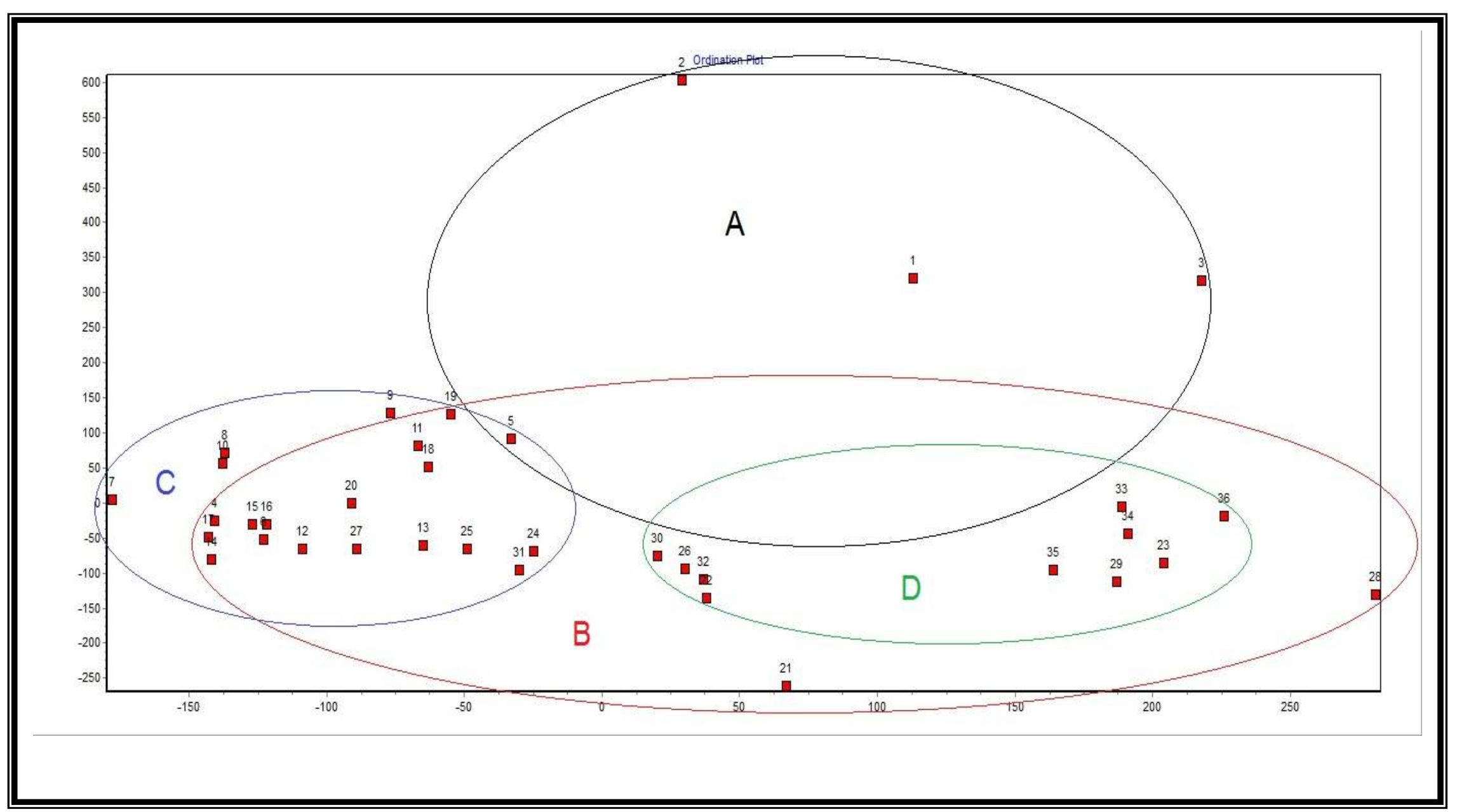

Figure (3): DCA ordination diagram of the four vegetation groups identified by TWINSPAN classification. 


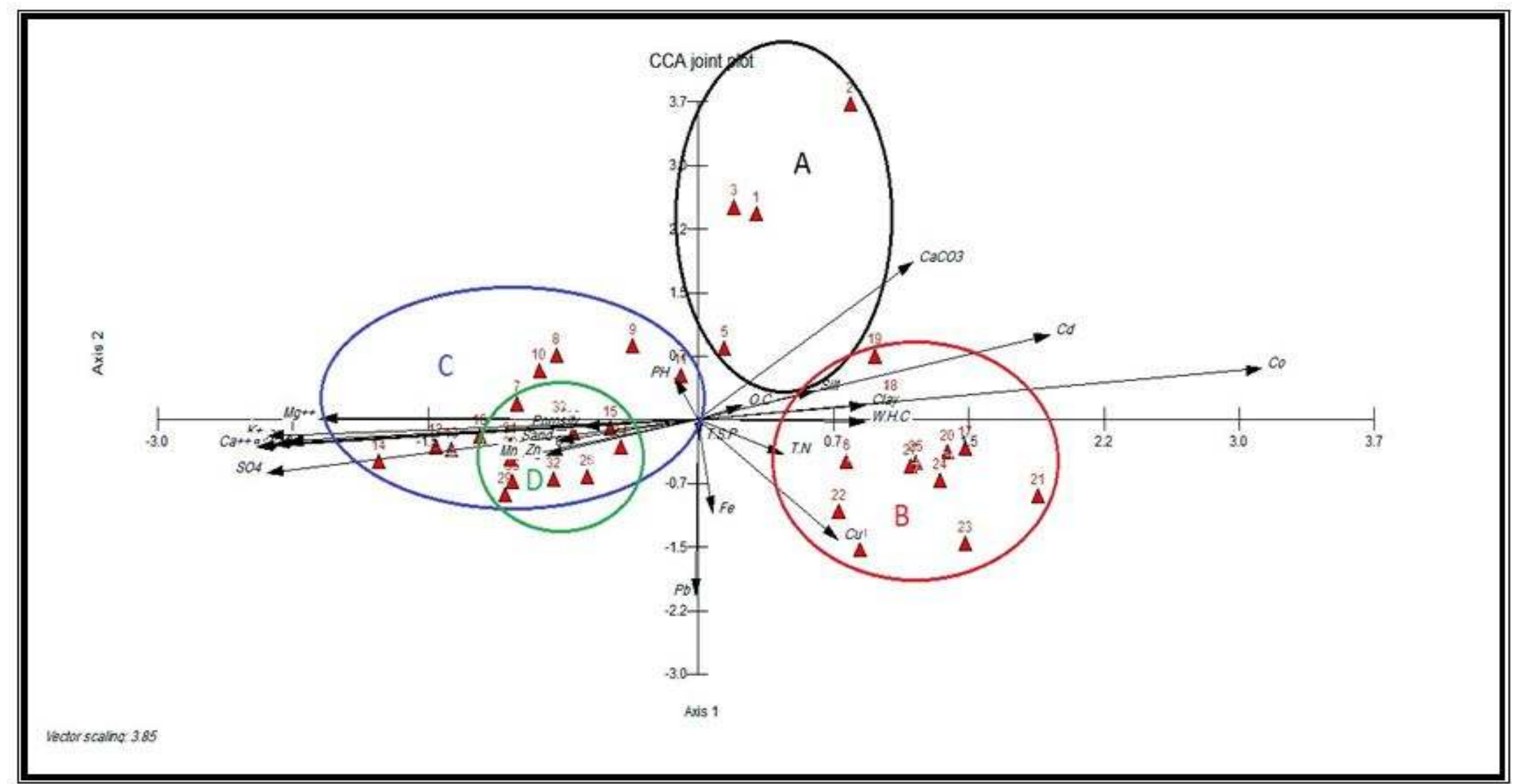

Figure (4): CCA-ordination biplot of the sampled stands and soil variables in the study area. 
Table (4): Mean values \pm standard errors of the soil variables representing the four vegetation groups obtained by TWINSPAN. MWHC: maximum water-holding capacity; MC: moisture content; OC: organic carbon; EC: electrical conductivity; TN: total nitrogen; TDP:total dissolved phosphorus.

\begin{tabular}{|c|c|c|c|c|}
\hline \multirow[b]{2}{*}{ Soil variable } & \multicolumn{4}{|c|}{ Vegetation group } \\
\hline & A & " B & $\mathbf{C}$ & D" \\
\hline Sand & $74.41 \pm 0.487$ & $71.48 \pm 2.19$ & $71.44 \pm 2.042$ & $76.46 \pm 1.040$ \\
\hline Silt & $13.78 \pm 0.148$ & $15.8 \pm 1.137$ & $16.38 \pm 1.390$ & $12.96 \pm 0.678$ \\
\hline Clay (\%) & $10.97 \pm 0.344$ & $12.72 \pm 1.075$ & $12.2 \pm 0.760$ & $10.56 \pm 0.383$ \\
\hline M.W.H.C & $34.62 \pm 0.730$ & $38.42 \pm 1.906$ & $37.9 \pm 1.366$ & $34.06 \pm 1.079$ \\
\hline Porosity & $37.79 \pm 1.566$ & $39.77 \pm 1.306$ & $43.2 \pm 1.700$ & $39.40 \pm 1.370$ \\
\hline O.C & $0.275 \pm 0.0332$ & $0.36 \pm 0.0419$ & $0.43 \pm 0.0549$ & $0.23 \pm 0.0295$ \\
\hline PH & $8.2 \pm 0.080$ & $8.13 \pm 0.0459$ & $8.22 \pm 0.0378$ & $8.1 \pm 0.051$ \\
\hline $\mathrm{EC}\left(\mathrm{mmhos} \mathrm{cm}^{-1}\right)$ & $1.61 \pm 0.252$ & $1.60 \pm 0.127$ & $2.162 \pm 0.077$ & $2.085 \pm 0.173$ \\
\hline $\mathrm{CaCO}_{3}$ & $4.46 \pm 0.285$ & $3.422 \pm 0.3108$ & $2.9 \pm 0.219$ & $2.75 \pm 0.310$ \\
\hline (\%) $\mathrm{Cl}^{-}$ & $5.1 \pm 0.772$ & $5.17 \pm 0.396$ & $6.70 \pm 0.193$ & $6.29 \pm 0.515$ \\
\hline $\mathrm{SO}_{4}^{--}$ & $1.47 \pm 0.278$ & $1.45 \pm 0.1570$ & $2.08 \pm 0.135$ & $2.18 \pm 0.213$ \\
\hline $\mathrm{HCO}_{3}^{-}$ & $1.67 \pm 0.240$ & $1.7 \pm 0.1342$ & $2.28 \pm 0.080$ & $2.19 \pm 0.203$ \\
\hline T.D.P & $6.31 \pm 0.145$ & $7.08 \pm 0.3210$ & $7.5 \pm 0.405$ & $6.70 \pm 0.236$ \\
\hline T.N & $33.28 \pm 0.306$ & $44.4 \pm 3.051$ & $46.56 \pm 3.229$ & $36.61 \pm 0.798$ \\
\hline $\mathrm{Na}^{+} \quad(\mathrm{mg} / 100 \mathrm{~g}$ dry soil $)$ & $5.24 \pm 0.77$ & $5.32 \pm 0.4002$ & $6.9 \pm 0.192$ & $6.59 \pm 0.491$ \\
\hline $\mathbf{K}^{+}$ & $0.37 \pm 0.066$ & $0.36 \pm 0.0327$ & $0.52 \pm 0.020$ & $0.47 \pm 0.0431$ \\
\hline $\mathrm{Ca}^{++}$ & $1.56 \pm 0.243$ & $1.58 \pm 0.1327$ & $2.15 \pm 0.081$ & $2.07 \pm 0.176$ \\
\hline $\mathbf{M g}^{++}$ & $1.05 \pm 0.205$ & $0.96 \pm 0.0938$ & $1.5 \pm 0.114$ & $1.27 \pm 0.194$ \\
\hline $\mathbf{F e}$ & $24.72 \pm 0.146$ & $26.13 \pm 0.5273$ & $26.05 \pm 0.185$ & $25.79 \pm 0.48$ \\
\hline Mn & $9.94 \pm 0.274$ & $10.51 \pm 0.455$ & $16.87 \pm 7.60$ & $13.37 \pm 0.25$ \\
\hline $\mathbf{Z n}$ & $15.9 \pm 0.096$ & $16.47 \pm 0.431$ & $17.02 \pm 0.256$ & $16.9 \pm 0.55$ \\
\hline $\mathrm{Cu}$ & $4.96 \pm 0.138$ & $6.2 \pm 0.194$ & $6.006 \pm 0.112$ & $5.3 \pm 0.318$ \\
\hline Co & $0.74 \pm 0.025$ & $0.77 \pm 0.029$ & $0.594 \pm 0.0148$ & $0.59 \pm 0.026$ \\
\hline Cd & $1.65 \pm 0.013$ & $1.67 \pm 0.0294$ & $1.613 \pm 0.041$ & $1.45 \pm 0.039$ \\
\hline $\mathbf{P b}$ & $7.49 \pm 0.191$ & $8.71 \pm 0.171$ & $0.210 \pm 8.7$ & $8.45 \pm 0.13$ \\
\hline
\end{tabular}

\section{DISCUSSION}

Egypt is located in arid or semi-arid regions, is facing a severe problem of feed supply shortage or high cost especially green summer fodder (Shaltout et al., 2009). The main causes of this problem involve population increase, frequent drought, limited renewable natural resources, limited cultivated lands with forages and expansion of urbanization at the expense of agricultural areas. This problem reduces the enhancement of animal/meat production. Therefore, calls for searching for other non-conventional novel alternative sources for feed become required and urgent (Elias, 2015).

The decline in forage in Egypt has been generally attributed to a number of factors such as scarce of rainfall and frequent drought, overgrazing of range shrubs and bushes for fuel and expansion of urbanization. The forage value of a consumed plant is the result of two main components: 1) palatability and voluntary intake by livestock, and 2) nutritive value i.e. chemical composition and digestibility (Le Houerou, 1980). At the same time, the flora of the Nile Delta and its coast is rich by many weeds, which seem to be promising economically. Some weeds can be used as forages, and /or agro-industrial raw materials and in drug industry (El- Halawany et al., 2008).

The natural plant life in the present investigation was composed of 79 plant species belonging to 70 genera and related to 26 taxonomic families. These species were classified into 44 annuals and 33 perennials. The life- form spectra of the recorded species mainly include therophytes $(55.69 \%)$, followed by crypto- phytes $(16.45 \%)$, then hemicryptophytes $(10.12 \%)$ and finally chamaephytes and phanerophytes $(8.80 \%$ each). This agrees more or less with the findings of (Mashaly, 2001\&2002) on the ecology of the Deltaic.

Mediterranean coast and (Abd El Aal, 2013) on the plant life in the different habitats in El-Behira Governorate. The dominance of therophytes is probably due to their short life cycle that enables them to resist the instability of the environmental condition. They have also an ability to set seeds without the need of pollinator visit (Baker, 1974). Also, the relative high frequency of cryptophytes as an active life-form in the study area could be related to certain features of both their growth habit and nature of soil. Most of the recorded cryptophytes are rhizomatous species; this is an advantage for successful growth and their distribution (Shaltout et al.,1995).

In the present study, the chorological affinities revealed that the Sahro-Sindian element was represented by 34 species which can be distinguished into 15 Biregional, 11 Pluriregional and eight Monoregional elements. Irano-Turanian element was represented by 21 species which can be classified into 11Pluriregional and 10 Biregional elements. EuroSiberian element was represented by 10 species which can be divided into eight Pluriregional and two Biregional elements. Sudano-Zambezian element was represented by nine species which distinguished into seven Biregional and two Pluriregional elements. Cosmopolitan element was represented by six species, Pantropical element by three species, Palaeotropical and Neotropical by one species each. This was Confir- 
med by (Mashaly et al., 2008, 2014 \&2015a\&bc), (ElHalawany et al., 2010) and (Abu-Zida et al.2015).

Phytosociologically, the vegetation that characterizes classification into four groups (A, B, C\& D). Each group comprises a number of sampled stands which are similar in terms of vegetation and characterized by dominant and / or codominant species as well as by a number of indicator species. Group A includes 21 species in 4 stands and it was codominant by Elymus farctus and Calligonum polygnoides no indicator species in this group was detected. Group B comprises 50 species in 13 stands and it was dominated by Hordeum murinum, the indicator species in this grpup include Lactuca serriola, Tamrix tetragyna, Lotus halophilus, Melilotus indicus and Rumex pictus. Group $\mathrm{C}$ includes 38 species in 11 stands and it was codominated by Stipagrostis lanata and Echinops spinosus, two indicator species in this group were Plantago squarrosa and Senecio glaucus. Group D comprises 43 species in 8 stands and it wsa codominated by Lycium schweinfurthii and Echinops spinosus, also two indicator species in this group were Atriplex halimus and Rumex pictus. The identified association in the present study might be coincide with numerous previous studies in various regions of Egypt (viz Shaltout and Sharf El Din. 1988), (Shaltout et al. 2008\&2010), (Mashaly, 2001\&2002), (El-Halawany et al., 2010), (Mashaly et al., 2014,2015 a,b\&d), (AbuZaida et al., 2015) and (Al-Barti, 2018).

The Detreanded Correspondence Analysis (DCA) ordination plot of the 36 stands on axes 1 and 2 exhibited that the four vegetation groups obtained by TWINSPAN classification were markedly undistinguishable and super-imposed, this may be due to the close similarities in the floristic composition of these vegetation groups. The ordination of the vegetation groups in the present study may be coincide with many previous studies in different regions of Egypt e.g (Taksera, 2014), (Yahia, 2015), (Elias, 2015), (Abdrabbu, 2016), (EL-Naggar, 2018) and (Al-Barati, 2018).

The correlation between vegetation and soil characteristics as indicated on the ordination diagram (CCAbiplot) revealed that sulphates, calcium, magnesium, potassium, cobalt, calcium carbonate, cadmium and copper were the most effective soil variables controlling the distribution and abundance of vegetation groups in the study area. These results are in agreement with other many previous studies on the vegetation-soil relationship in Egypt as (Sharf EL-Din and Shaltout. 1985), (Abd El- Ghani and Amer. 2003), (Hegazy et al., 2004\&2006), (Mashaly et al., 2011,2012\&2013), (Salama et al., 2013\&2015) and (El-Naggar, 2018).

In conclusion, the present work is attempt to investigate the floristic composition and vegetation analysis of the flora associated with some grasses growing naturally in the coast of Nile Delta region which can be used as renewable natural resources.

\section{REFERENCES}

ABD EL- AAL, M. 2013. Plant life in the Different Habitats in El-Behira Governorates, Egypt. Ph.D. Thesis, Fac.Sci, Mansoura University.
ABD EL-GHANI, M.M.AND W.M. AMER. 2003. Soil Vegetation Relationships in a Coastal Desert Plain of Southern Sinai, Egypt. Journal of Arid Environments, 55:607-628.

ABDRABBU, H.S.2016. Weed ecology in reclaimed lands in Eastern and Western sides of Qena Governorates, Egypt.Ph.D. Thesis, Fac.Sci, South Valley University, Qena.

ABU AL-IZZ, M.S.1971. Land Forms of Egypt. The American University in Cairo Press. Dar Al Maaref, Cairo.

ABU-ZIADA, M.E., I.A. MASHALY, A.M. ELGAWAD AND M.R. DEWEEB. 2015. Floristic Features of three Plantago species Communities in the Nile Delta, Egypt. Journal of Environmental Sciences, Mansoura University, 44 (3) :427-441.

AL-BARATI, S.A. 2018. Weed Communities of Orchards in Nile Delta, Egypt: Ecology and Economic Potentialities. Ph.D. Thesis, Fac.Sci., Mansoura University.

ALLEN, S.E., H.M. GRIMSHAW, J.A. PARKINSON, C. QUARMBY AND J.D. ROBERTS. 1974. Chemical Analysis of Ecological Materials. Blackwell Scientific Publ.Osney, Oxford, London.

APHA (American Public Health Association) (1998). Standard Methods for the Examination of Water and Waste Water, $19^{\text {th }}$ Edition. Water Pollution Control Federation, Washington, D.C..

Ayyad, M. A. AND R. El-GHAREEB. 1984. Habitats and Plant Communities of the Northeastern Desert of Egypt. Communications in Agriscience and Development Research, 7,No.60.

BAKER, H.G. 1974. The Evolution of weeds. Annual Review of Ecology and Systematics, 5 : 1-24.

BOULOS, L.1999, 2000, 2002\& 2005. Flora of Egypt. Vols.1,2,3\&4.Al Hadara Publishing, Cairo, Egypt.

BOULOS, L. 2009. Flora of Egypt Checklist. Al Hadara Publishing, Cairo, Egypt. CANFIELD, R. 1941. Application of the line Interception Method in Sampling Range Vegetation. Jornal. Forestry ,39:288-394.

EL-GABALY, M.M., I.M. GEWAFEL, M.N. HASSAN, AND B.G. ROZANON. 1969a. Soil and soil regions of United Arab Republic. Research Bulletin of Alexandria University, 21:1-28.

EL-GABALY, M.M., I.M. GEWAFEL, M.N. HASSAN AND B.G. ROZANON. 1969 b. Soil Map and land Resources of the United Arab Republic. Research Bulletin of Alexandria University, 22:1-14.

EL-HALAWANY, E.F., I.A. MASHALY, M.E. ABUZIADA AND M. EL-AAL. 2010. Habitat and plant life in Dakahlyia Governorate ,Egypt. Journal of Environmental Sciences, Mansoura University, 39 (1): 83-108.

EL-HALAWANY, E.F. , I.A. MASHALY, I.E. ELHABASHY AND G. OMER. 2008. Autecology and Fodder Potentialities of Some Species in the Nile Delta of Egypt. Journal of Environmental Sciences, Mansoura University, 36: 29-49.

EMBERGER, L.1955. Une Classification Biogegraphiques Des Climates Recueil Des Travaux De La Faculte De Science dl' Universite Mont. Pollier, 
Fascicule, 7:3-43.

FEINBRUN-DOTHAN, N. 1978\& 1986. Flora Palaestina . Parts. $3 \& 4$. The Israel Academy of Sciences and Humanities, Jerusalem.

HASSIB, M.1951. Distribution of Plant communities in Egypt. Faculty of Science Bulletin, Fouad University, 29 : 259-261.

HEGAZY, A.K., G.M. FAHMY, M.I. ALI AND H.N. GOMMA, 2004. Vegetation Diversity in Natural and Agro-ecosystems of Arid lands. Community Ecology, 5 (2): 163-176.

HEGAZY, A.K., S. MUSSA, AND H.F. FARRAG, 2008. Invasive Plant Communities in the Nile Delta Coast . Global Journal of Environmental Research, 2:53-61.

HENDERSON, P.A. AND R.M. SEABY, 1999. Community Analysis Package (cap) Version 1.2.Pisces Conservation Ltd. IRC House, UK.

JACKSON, M.L.1967.Soil Chemical Analysis. Constable and Co.LTD.London, UK.

KOPPEN, S. W.1931.Grundriss der klimakunde, w. de Gruyter, Berlin

LE HOUEROU, H.N.1980.Chemical Composition and Nutritive Value of in Tropical West Africa. Browse in Africa, the Current state of knowledge. Le Houerou, H.N.(ed.), ILCA, Addis Ababa:261-289.

MASHALY, I.A.1987.Ecological and Floristic Studies of Dakahlia-Damietta region . PhD. Thesis, Fac.Sci., Mansoura University

MASHALY, I.A.2001.Contribution to the Ecology of the Deltaic Mediterranean Coast, Egypt. Online Journal of Biological. Sciences, 1(7) : 628-635.

MASHALY, I.A.2002. Ecological studies on Zygophyllum aegyptium in the Deltaic Mediterranean coast of Egypt. Pakistan Journal of Biological Sciences, 5(2) : 152-160.

MASHALY, I.A., M. ABD EL-AAL, AND N.K. DAWOOD. 2015 d. Floristic Composition and Vegetation Analysis and Species Diversity of Some Brassica species Associates in North Nile Delta region, Egypt. Catrina, 14 (1) :43-53.

MASHALY, I.A., M.E. ABU-ZIADA, Y.A. ELAMIER, AND S.M. KHALIFA. 2015 c. Floristic Characterization of the Plant Communities Aassociated with Some Species of Genus Euphorbia in Egypt. Journal of Environmental Sciences, Mansoura University, 44 (3) : 525-548.

MASHALY, I.A., M.E. ABU-ZIADA, Y.A. ELAMIER AND R.M. KHORSHIED. 2015 a. Ecological Study on Two Species of Genus Rumex in the Nile Delta, Egypt. Journal of Environmental Sciences, Mansoura University, 44 (2): 403-425.

MASHALY, I.A., Y.A. EL-AMIER, AND F.O. ELKARNAWDY. 2015 b. Floristic Characterization of the Plant Cover Associated with spiny Leaved Species in Family Asteraceae in Nile Delta coast . Journal of Environmental Sciences, Mansoura University, 44 (2) : 345-359.

MASHALY, I.A., I.S. EL-HABASHY, H.S. ALDESUQUY, E.F. EL-HALWANY AND N.R. TAKSERA. 2014. Ecological study on genus Echinochloa in the North Nile Delta, Egypt. Journal of Environmental Sciences, Mansoura University, 43 (3) : 349-367.

MASHALY, I.A., I.E. EL-HABASHY, E.F. ELHALAWANY, AND G. OMAR. 2012. Weed Plant Communities in the Nile Delta of Egypt.III. Cultivated land habitat. Egyptian Journal of Botany, 52 (1) : 1-26.

MASHALY, I.A., I.S. EL-HABASHY, E.F. ELHALAWANY, AND G. OMAR. 2008. Habitats and Plant Communities in the Nile Delta of Egypt. I. Deltaic Mediterranean Coastal Habitat. Pakistan Journal of Biological Sciences, 12 (12):885-895.

MASHALY, I.A., E.F. EL- HALWANY AND A.M. ABD EL-GAWAD. 2011.Ecology of Weeds and Invasive Plant Species in Newly Reclaimed Areas in the Nile Delta, Egypt. Journal of Environmental Sciences, Mansoura University, 40 (1) : 69-90.

MASHALY, I.A., E.F. EL- HALWANY, M.E. ABUZIADA, AND M. ABD EL- AAL, 2013. Vegetation Soil Relationship in the Cultivated land Habitat in El-Behira Governorate, Egypt. Journal of Environmental Sciences, 42 (4):607-623.

MUELLER-DOMBOIS, D. AND H. ELLENBERG. 1974. Aims and Methods of Vegetation Ecology. John Wiley and Sons, New York, Chichester, Brisbane, Toronto.

PIERCE, W.C., E.L. HAENISCH, and D.T. SAWYER. 1958. Quantitative Analysis. John Wiley, New York: 49-51.

PIPER, C.S.1947. Soil and plant analysis. Inter Science Publishers, Inc, New York, USA.

PIRIE, N.W. 1955. Protein. In: Modern Methods of Planrt Analysis IV, 23, Springer Verlage, Berlin.

RANGACHARI, K. AND C. TADULINGAM, 2007. A Handbook of Some South Indian Grasses. Gutenberg Project.

SALAMA, F.M., M.M. ABD EL-GHANI AND N. ELTAYEH, 2013. Vegetation and Soil Relationships in the Inland Wadi Ecosystem of Central Eastern Desert, Egypt. Turkish Journal of Botany, 37 : 489 498.

SALAMA, F.M., M.M., ABD EL-GHANI, M. GADALLAH, S. EL-NAGGAR, AND A. AMRO. 2015.Diversity and Response of Plant Functional Groups to Soil Variables in the Arid Desert landscape of Southern. Egypt. Journal of Biodiversity and Ecological Science, 5(1): 24-39.

SESTINI, G. 1976. Geomorphology of the Nile Delta, UNESCO Proceeding of Seminar on Nile Delta Sedimentology, Alexandaria.

SHALTOUT, K.H., AND A. SHARAF EL-DIN. 1988. Habitat Types and Plant Communities along a Transect in the Nile Delta region. Feddes Repertorium, 99: 153-162.

SHALTOUT, K.H., H.F. EL-KADY AND Y.M. ALSODANY. 1995. Vegetation Analysis of the Mediterranean region of Nile Delta. Vegetatio, 116 (1):73-80.

SHALTOUT, K.H., A.A. EL-KEBLAWY, AND T.M. MOUSA. 2008. Evaluation of the Range Plants Quality and Palatability for Camel Grazing in the United Arab Emirates. Journal of Camelid Sciences, 1: 1-13. 
SHALTOUT, K.H., A. SHARAF EL-DIN, AND D.A. AHMED. 2010. Plant life in the Nile Delta. Tanta University Press, Tanta, Egypt.

SHARAF EL-DIN, A. AND K.H. SHALTOUT. 1985. On the Phytosociology of Wadi Araba in the Eastern Desert of Egypt. Proc. Egypt. Bot. Soc. IV Ismailia Conf.,:1311-1317.

SHUKLA, R.S. AND P.S. CHANDEL. 1989. Plant Ecology and Soil Science. S.C Hand \& Company LTD. Ram Nagar, New Delhi, India.

TÄCKHOLM, V. 1974. Students' Flora of Egypt. $2^{\text {nd }}$ ed. Cairo University Press. Cairo, Egypt.

TAKSERA, N.R.2014. Ecological and Physiological Study on genus Echinochloa In North Nile Delta of Egypt. Ph.D. Thesis, Fac. Sci., Mansoura
University. TER- BRAAK, C.J. 2003. CANOCO, Version 4.52. Wageningen, the Netherlands.

THORNTHWAITE, C.W.1948. An Approach Towards a National Classification of Climate. Geographical Review, 38:55-94. UNESCO,1977. Map of world distribution of arid regions. MAB Technical Notes, 7.

YAHIA, A.A.2015. Ecological Study and Economic Potentialities of Some Species of Genus Zygophyllum in Egypt. M.Sc. Thesis, Fac.Sci, Mansoura University.

ZOHARY, M.1966\&1972. Flora Palestina. Parts 1 and 2. The Israel Academy of Sciences and Humanities, Jerusalem.

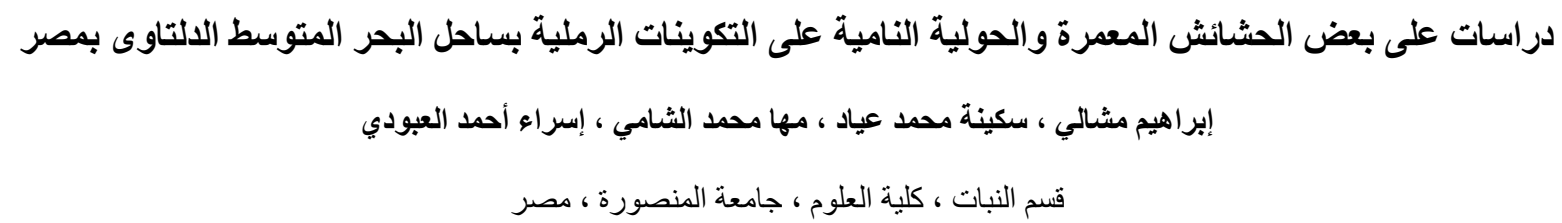

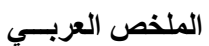

تهدف هذه الدراسه الى اجر اءدراسات بيئية تفصيلية على بعض الحشائش الناميه بريا بساحل الدلتا المطل على البحر المنوسط وتشمل حشائش سفسوف وشعير بري وشعير الفار وشفشوف والجازوف وتهدف هذه الدراسة إلى ما يلي:اولا در اسة السمات الفلورية والتي تشتمل تسجيل الانواع النباتية و المظاهر المفصلية (Life-span) وتصنيف الطرز الحياتية (Life-form) وتحليل ونيل العناصر الفلورية (Chorotypes) للأنواع النباتية المصاحبة للحشائش المختارة بمنطقه الدراسة. ثانيا تحليل كمي لتركيب ووفره المجتمعات النباتيه المصاحبه لانو اع الحشائش المختاره باستخدام طرق التحليل المتعددة للتصنيف و التسلسل (Class.\& ordina). ثالثا تحليل التغيرات في عوامل التربة وذلك لتحديد اهم العوامل المؤثره في نوزيع ووفرة المجتمعات النباتبة المرافقة للحشائش

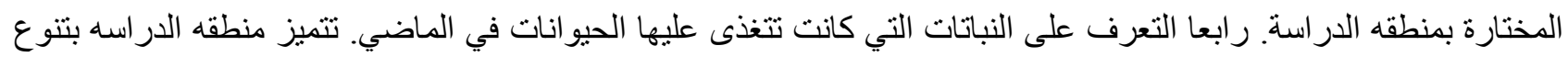
عالي في الانواع النباتية حيث ان العدد الكلي الذي تم تسجيله في عدد 36 واققفة كان 79 نو عا تم تقسيمها الي 44 نوعا من طر از الحوليات و13 نوعا من طراز المختفيات وثمانية انواع من طر از شبة المختفيات وسبعه انو اع لكل من طر ازي النباتات الظاهرة و النباتات الخشبية، كما اتضح ان النباتات المسجلة تتبع 70 جنسا و26عائله نباتيه وظهر ان اكثر العائلات وفرة بالأنواع النباتية

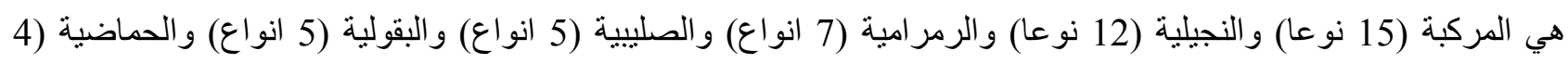

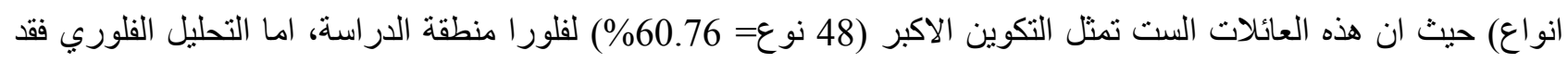

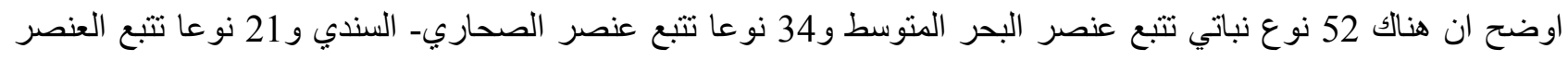
الاير اني- الاناضول و10 انواع تتبع العنصر الاوربي_ السيبري و9 انواع تتبع العنصر السوداني_ الزمبزي و6 انواع تتبع العنصر العالمي و3 انواع تتبع العنصر الاستوائي ونوع واحد لكل العنصر الاستوائي القديم والعصر الاستوائي الحديث و المنزر عة أو المستأنسة. وبيئيا فقد تم تحليل الغطاء النباتي المر افق لأنواع الحشائش المختارة باستخدام تقنيات التحاليل المتعددة (التصنيف و التسلسل) اما تقنية التصنيف فقد تم تطبيق برنامج التصنيف ثنائي الاتجاه (TWINSPAN) نم تطبيق برنامج التوزيع الطابقي الانعكاسي (DCA) وبرنامج التوزيع التطابقي الكنسي (CCA). وباستخدام وتطبيق برامج 
التصنيف ثنائى الاتجاه لقيم الاهميه لعدد 79 نوعا نباتيا مسجله داخل 36 موقعا فقد ادى الى التعرف على اربعه مجمو عات نباتيه سميت كما يلي:مجمو عة (ا) يسودها كل من نباتي الجازوفــ الارطة؛ مجموعة (ب) يسودها نبات شعير بري؛ مجموعة

$$
\text { (ج) يسودها نباتي شفشوفـ الكداد؛ مجموعة (د) يسودها نبات العوسج- الكداد. }
$$

بتطبيق برنامج التوزيع التطابقى العكسى (DCA) فقد أوضح ان المجموعات النباتية الاربعة منر اكبة ومتداخلة مع بعضها بوضوح و غير منفصلة، اما عند نطبيق برنامج التطابق الكنسي (CCA) لايضاح العلاقة بين المجموعات النباتية وعو امل التربة فقد اتضح ان كل من الكبريتات و الكالسيوم و الماغنسيوم و البوتاسيوم و و الكوبلت وكربونات الكالسيوم و الكادميوم والنحاس كانت

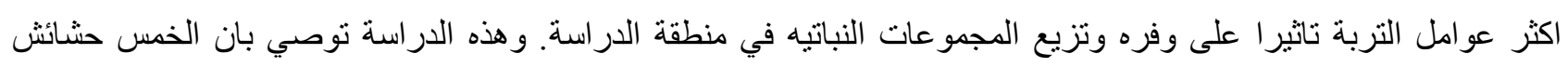

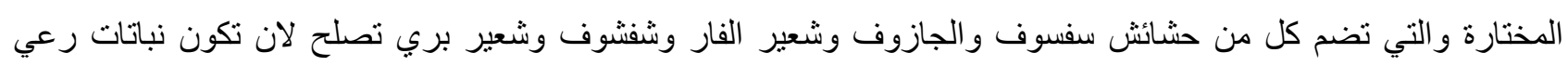
للحيو انات، وايضا فان تحليل حفريات حبوب اللقاح فقد اثبتت ان ليست افر اد العائلة النجيلية وحدها تصلح كاعلاف ولكن ايضا هناك عائلات نباية اخرى تصلح في هذا الصدد. 